\title{
EXISTENCE OF POSITIVE SOLUTIONS FOR SOME NONLINEAR ELLIPTIC PROBLEMS IN UNBOUNDED DOMAINS OF $\mathbb{R}^{n}$
}

\author{
NOUREDDINE ZEDDINI
}

Received 26 May 2005; Accepted 1 August 2005

This paper deals with a class of nonlinear elliptic equations in an unbounded domain $D$ of $\mathbb{R}^{n}, n \geq 3$, with a nonempty compact boundary, where the nonlinear term satisfies some appropriate conditions related to a certain Kato class $K^{\infty}(D)$. Our purpose is to give some existence results and asymptotic behaviour for positive solutions by using the Green function approach and the Schauder fixed point theorem.

Copyright (c) 2006 Noureddine Zeddini. This is an open access article distributed under the Creative Commons Attribution License, which permits unrestricted use, distribution, and reproduction in any medium, provided the original work is properly cited.

\section{Introduction}

In this paper, we are concerned with the following nonlinear elliptic equation

$$
\Delta(u)+f(\cdot, u)=0 \quad \text { in } D
$$

(in the sense of distributions) with some boundary values (see problems (1.8), (1.15) below), where $D$ is an unbounded domain in $\mathbb{R}^{n}(n \geq 3)$ with a nonempty compact boundary.

Numerous results are obtained for (1.1), in both bounded and unbounded domains $D \subset \mathbb{R}^{n}$ with different boundary conditions (see, e.g., $[2,5-9,11,12]$ and the reference therein).

Our aim in this paper is to undertake a study of (1.1) when the nonlinear term $f(x, t)$ satisfies some appropriate conditions related to a certain Kato class of functions $K^{\infty}(D)$ and to answer the questions of existence and asymptotic behaviour of positive solutions.

Our tools are based essentially on some inequalities satisfied by the Green function $G_{D}(x, y)$ of $(-\Delta)$ in $D$ which allow to some properties of functions belonging to the class $K^{\infty}(D)$ introduced in [1] as the following definition. 
2 Positive solutions on some exterior domains

Definition 1.1. A Borel measurable function $q$ in $D$ belongs to the class $K^{\infty}(D)$ if $q$ satisfies the following conditions:

$$
\begin{aligned}
& \lim _{\alpha \rightarrow 0}\left(\sup _{x \in D} \int_{(|x-y| \leq \alpha) \cap D} \frac{\rho_{D}(y)}{\rho_{D}(x)} G_{D}(x, y)|q(y)| d y\right)=0, \\
& \lim _{M \rightarrow \infty}\left(\sup _{x \in D} \int_{(|y| \geq M) \cap D} \frac{\rho_{D}(y)}{\rho_{D}(x)} G_{D}(x, y)|q(y)| d y\right)=0,
\end{aligned}
$$

where $\rho_{D}(x)=\delta_{D}(x) /\left(1+\delta_{D}(x)\right)$ and $\delta_{D}(x)$ denotes the euclidien distance from $x$ to the boundary of $D$.

We will often refer in this paper to the bounded continuous solution $\mathrm{Hg}$ of the Dirichlet problem

$$
\begin{gathered}
\Delta w=0 \quad \text { in } D, \\
w / \partial D=g, \\
\lim _{|x| \rightarrow \infty} w(x)=0,
\end{gathered}
$$

where $g$ is a nonnegative bounded continuous function in $\partial D$.

We also refer to the Green potential of a measurable nonnegative function $f$, defined in $D$ by

$$
V f(x)=\int_{D} G_{D}(x, y) f(y) d y .
$$

Our paper is organized as follows. Our existence results are proved in Sections 3 and 4. In Section 2, we collect and improve some preliminary results about the Green function $G_{D}$ and the class $K^{\infty}(D)$. In Section 3, we establish an existence result for (1.1) where a singular term and a sublinear term are combined in the nonlinearity $f(x, t)$.

The pure singular elliptic equation

$$
\Delta u+p(x) u^{-\gamma}=0, \quad \gamma>0, x \in D \subset \mathbb{R}^{n}
$$

has been extensively studied for both bounded and unbounded domain $D$. We refer to ([5-9] and the references therein) for various existence and uniqueness results related to solutions for (1.5).

For more general situations and when $D$ is an unbounded domain with a nonempty compact boundary Bachar et al. showed in [1] that the following problem:

$$
\begin{gathered}
\Delta u+\varphi(x, u)=0 \quad \text { in } D, \\
u / \partial D=0, \\
\lim _{|x| \rightarrow \infty} u(x)=0,
\end{gathered}
$$

admits a unique positive solution if $\varphi$ is a nonnegative measurable function on $(0, \infty)$, which is nonincreasing and continuous with respect to the second variable and for each $c>0$, the function $\varphi(\cdot, c) \in K^{\infty}(D)$. 
On the other hand, (1.1) with a sublinear term $f(\cdot, u)$ have been studied in $\mathbb{R}^{n}$ by Bresis and Kamin [2]. Indeed, the authors proved the existence and uniqueness of a positive solution for the problem

$$
\begin{gathered}
\Delta u+\rho(x) u^{\alpha}=0 \quad \text { in } \mathbb{R}^{n}, \\
\liminf _{|x| \rightarrow \infty} u(x)=0,
\end{gathered}
$$

with $0<\alpha<1$ and $\rho$ is a nonnegative measurable function satisfying some appropriate conditions.

In the third section, we combine a singular term and a sublinear term in the nonlinearity. Indeed, we consider the following boundary value problem

$$
\begin{gathered}
\Delta u+\varphi(x, u)+\psi(\cdot, u)=0 \quad \text { in } D \text { (in the sense of distributions), } \\
u>0 \quad \text { in } D, \\
u / \partial D=0, \\
\lim _{|x| \rightarrow \infty} u(x)=0,
\end{gathered}
$$

where $\varphi$ and $\psi$ are required to satisfy the following hypotheses.

$\left(\mathrm{H}_{1}\right) \varphi$ is a nonnegative Borel measurable function on $D \times(0, \infty)$, continuous and nonincreasing with respect to the second variable .

$\left(\mathrm{H}_{2}\right) \forall c>0, x \rightarrow \varphi(x, c \theta(x)) \in K^{\infty}(D)$, where $\theta(x)=\delta_{D}(x) /(1+|x|)^{n-1}$.

$\left(\mathrm{H}_{3}\right) \psi$ is a nonnegative Borel measurable function on $D \times(0, \infty)$, continuous with respect to the second variable such that there exist a nontrivial nonnegative function $p$ and a nonnegative function $q \in K^{\infty}(D)$ satisfying for $x \in D$ and $t>0$

$$
p(x) f(t) \leq \psi(x, t) \leq q(x) g(t)
$$

where $f$ is a measurable nondecreasing function on $[0, \infty)$ satisfying

$$
\lim _{t \rightarrow 0^{+}} \frac{f(t)}{t}=+\infty
$$

and $g$ is a nonnegative measurable function locally bounded on $[0, \infty)$ satisfying

$$
\limsup _{t \rightarrow \infty} \frac{g(t)}{t}<\frac{1}{\|V q\|_{\infty}}
$$

By using a fixed point argument, we will state the following existence result.

Theorem 1.2. Assume $\left(H_{1}\right)-\left(H_{3}\right)$. Then the problem (1.8) has a positive solution $u \in$ $C_{0}(D)$ satisfying for each $x \in D$

$$
a \theta(x) \leq u(x) \leq V(\varphi(\cdot, a \theta))(x)+b V q(x)
$$

where $a, b$ are positive constants. 
4 Positive solutions on some exterior domains

Note that in [11] Mâagli and Masmoudi studied the case $\varphi=0$, under similar conditions to those in $\left(\mathrm{H}_{3}\right)$. Indeed the authors gave an existence result for

$$
\Delta u+\psi(\cdot, u)=0, \quad \text { in } D
$$

with some boundary conditions, where $D$ is an unbounded domain in $\mathbb{R}^{n}(n \geq 2)$ with a compact nonempty boundary.

Typical examples of nonlinearities satisfying $\left(\mathrm{H}_{1}\right)-\left(\mathrm{H}_{3}\right)$ are

$$
\begin{gathered}
\varphi(x, t)=p(x)(\theta(x))^{\gamma} t^{-\gamma}, \quad \text { for } \gamma \geq 0, \\
\psi(x, t)=p(x) t^{\alpha} \log \left(1+t^{\beta}\right), \quad \text { for } \alpha, \beta \geq 0 \text { such that } \alpha+\beta<1,
\end{gathered}
$$

where $p$ is a nonnegative function in $K^{\infty}(D)$.

In Section 4, we consider the nonlinearity $f(x, t)=-\varphi(x, t)$ and we use a fixed point argument to investigate an existence result for (1.1). More precisely we fix a nonnegative function $\xi$ continuous on $\partial D$ and we consider the following problem:

$$
\begin{gathered}
\Delta u=\varphi(x, u) \quad \text { in } D(\text { in the sense of distributions }) \\
u / \partial D=\xi \\
\lim _{|x| \rightarrow \infty} u(x)=\lambda \geq 0,
\end{gathered}
$$

where $\varphi: D \times[0, \infty) \rightarrow[0, \infty)$ is a Borel measurable function satisfying the following hypotheses:

$\left(\mathrm{H}_{4}\right) \varphi$ is continuous and nondecreasing with respect to the second variable,

$\left(\mathrm{H}_{5}\right) \varphi(x, 0)=0 ; \forall x \in D$,

$\left(\mathrm{H}_{6}\right) \forall c>0, \varphi(\cdot, c) \in K^{\infty}(D)$.

Under these hypothesis, we prove the following theorem.

Theorem 1.3. Assume $\left(H_{4}\right)-\left(H_{6}\right)$. Then the problem (1.15) has a unique nontrivial nonnegative solution $u \in C_{b}(D)$ satisfying

$$
0 \leq \lambda h(x)+H \xi(x)-u(x) \leq V \varphi(\cdot, c)(x) ; \quad \forall x \in D
$$

where $h$ is the harmonic function given by

$$
h(x)=1-H 1(x) .
$$

Remark 1.4 (see [3, page 116]). If we suppose further that there exists $\alpha \in(0,1)$ such that $\varphi$ is locally $\alpha$-hölder continuous on $D \times[0, \infty)$, then the solution $u$ of the problem $(1.15)$ is in $C^{2+\alpha}$ loc $(D)$.

As consequence of the preceding theorem we prove the following corollary.

Corollary 1.5. Let $a:[0, \infty) \rightarrow[0, \infty)$ be a continuous function. Assume that $\varphi$ is a locally hölder continuous function satisfying $\left(H_{4}\right)-\left(H_{6}\right)$ and let $\xi$ be a nontrivial nonnegative 
continuous function on $\partial D$. Then the following problem:

$$
\begin{gathered}
\Delta u+a(u)|\nabla u|^{2}=\varphi(\cdot, u) \quad \text { in } D \\
u=\xi \quad \text { on } \partial D \\
\lim _{|x| \rightarrow \infty} u(x)=\lambda \geq 0
\end{gathered}
$$

has a unique nontrivial nonnegative bounded solution $u \in C^{2}(D)$.

In order to simplify our statements, we define some convenient notations.

Notations. Throughout this paper, we will adopt the following notations.

(i) $D$ is an unbounded domain in $\mathbb{R}^{n}(n \geq 2)$ such that the complementary of $\bar{D}$ in $\mathbb{R}^{n}, \bar{D}^{c}=\bigcup_{j=1}^{d} D_{j}$ where $D_{j}$ is a bounded $C^{1,1}$-domain and $\bar{D}_{i} \cap \bar{D}_{j}=\varnothing$, for $i \neq j$.

(ii) $C_{b}(D)=\{f \in C(D): f$ is bounded in $D\}$.

(iii) $C_{0}(D)=\left\{f \in C(D): \lim _{x \rightarrow z \in \partial D} f(x)=\lim _{|x| \rightarrow \infty} f(x)=0\right\}$.

We note that $C_{b}(D)$ and $C_{0}(D)$ are two Banach spaces endowed with the uniform norm

$$
\|f\|_{\infty}=\sup _{x \in D}|f(x)|
$$

(iv) For $x \in D$, we denote by

$$
\lambda_{D}(x)=\delta_{D}(x)\left(\delta_{D}(x)+1\right) .
$$

(v) Let $f$ and $g$ be two positive functions on a set $S$.

We denote $f \sim g$, if there exists a constant $c>0$ such that

$$
\frac{1}{c} g(x) \leq f(x) \leq c g(x) \quad \forall x \in S .
$$

We denote $f \preceq g$, if there exists a constant $c>0$ such that

$$
f(x) \leq \operatorname{cg}(x) \quad \forall x \in S .
$$

(vi) We recall that if $f \in L_{\text {loc }}^{1}(D)$ and $V f \in L_{\text {loc }}^{1}(D)$, then we have in the distributional sense (see [3, page 52])

$$
\Delta(V f)=-f \quad \text { in } D .
$$

(vii) For each $q \in B^{+}(D)$ such that $V(q)<\infty$, we denote by $V_{q}$ the unique Kernel which satisfies the following resolvent equation (see [10])

$$
V=V_{q}+V_{q}(q V)=V_{q}+V\left(q V_{q}\right) \text {. }
$$

(viii) Let $f \in \mathscr{B}^{+}(D)$ such that $V f<\infty$. We recall that for each $x \in D$, the function $t \rightarrow V_{t q} f(x)$ is completely monotone on $[0,+\infty)$.

(ix) Let $a \in \mathbb{R}^{n} \backslash \bar{D}$ and $r>0$ such that $\overline{B(a, r)} \subset \mathbb{R}^{n} \backslash \bar{D}$. 
6 Positive solutions on some exterior domains

Then we have

$$
\begin{gathered}
G_{D}(x, y)=r^{2-n} G_{(D-a) / r}\left(\frac{x-a}{r}, \frac{y-a}{r}\right), \quad \text { for } x, y \in D, \\
\delta_{D}(x)=r \delta_{(D-a) / r}\left(\frac{x-a}{r}\right), \quad \text { for } x \in D .
\end{gathered}
$$

So without loss of generality, we may suppose throughout this paper that $\overline{B(0,1)} \subset$ $\mathbb{R}^{n} \backslash \bar{D}$. Moreover, we denote by $D^{*}$ the open set given by

$$
D^{*}=\left\{x^{*} \in B(0,1): x \in D \cup\{\infty\}\right\},
$$

where $x^{*}=x /|x|^{2}$ is the Kelvin inversion from $D \cup\{\infty\}$ onto $D^{*}$. Then, (see [1]), we have for $x, y \in D$,

$$
G_{D}(x, y)=|x|^{2-n}|y|^{2-n} G_{D^{*}}\left(x^{*}, y^{*}\right)
$$

\section{Properties of the green function and the class $K^{\infty}(D)$}

In this section, we recall and improve some results concerning the Green function $G_{D}(x, y)$ and the Kato class $K^{\infty}(D)$, which are stated in [1].

3G-Theorem. There exists a constant $C_{0}>0$ depending only on $D$ such that for all $x, y$ and $z$ in $D$

$$
\frac{G_{D}(x, z) G_{D}(z, y)}{G_{D}(x, y)} \leq C_{0}\left(\frac{\rho_{D}(z)}{\rho_{D}(x)} G_{D}(x, z)+\frac{\rho_{D}(z)}{\rho_{D}(y)} G_{D}(y, z)\right) .
$$

Proposition 2.1. On $D^{2}$ (i.e., $x, y \in D$ ), we have

$$
\begin{aligned}
G_{D}(x, y) & \sim \frac{1}{|x-y|^{n-2}} \min \left(1, \frac{\lambda_{D}(x) \lambda_{D}(y)}{|x-y|^{2}}\right), \\
& \frac{\rho_{D}(y)}{\rho_{D}(x)} G_{D}(x, y) \preceq\left(\delta_{D}(y)\right)^{2}, \\
& \frac{\delta_{D}(x) \delta_{D}(y)}{|x|^{n-1}|y|^{n-1}} \preceq G_{D}(x, y) .
\end{aligned}
$$

Moreover, for $M>1$ and $r>0$ there exists a constant $C>0$ such that for each $x \in D$ and $y \in D$ satisfying $|x-y| \geq r$ and $|y| \leq M$, we have

$$
G_{D}(x, y) \leq C \frac{\rho_{D}(x) \rho_{D}(y)}{|x-y|^{n-2}} .
$$

In the sequel, we use the notation

$$
\begin{aligned}
& \|q\|_{D}=\sup _{x \in D} \int_{D} \frac{\rho_{D}(y)}{\rho_{D}(x)} G_{D}(x, y)|q(y)| d y, \\
& \alpha_{q}=\sup _{x, y \in D} \int_{D} \frac{G_{D}(x, z) G_{D}(z, y)}{G_{D}(x, y)}|q(z)| d z .
\end{aligned}
$$


It is shown in [1] that

$$
\text { If } q \in K^{\infty}(D), \quad \text { then }\|q\|_{D}<\infty \text {. }
$$

Now, we remark that from the $3 \mathrm{G}$-theorem we have

$$
\alpha_{q} \leq 2 C_{0}\|q\|_{D}
$$

where $C_{0}$ is the constant given in the $3 \mathrm{G}$-theorem.

Proposition 2.2. For any nonnegative superharmonic function $v$ in $D$ and any $q \in K^{\infty}(D)$, we have

$$
\int_{D} G_{D}(x, y) v(y)|q(y)| d y \leq \alpha_{q} v(x), \quad \forall x \in D .
$$

Proof. Let $v$ be a positive superharmonic function in $D$. Then by ([13, Theorem 2.1, page $164])$, there exists a sequence $\left(f_{k}\right)_{k}$ of positive measurable functions in $D$ such that the sequence $\left(v_{k}\right)_{k}$ defined on $D$ by

$$
v_{k}(y):=\int_{D} G_{D}(y, z) f_{k}(z) d z
$$

increases to $v$.

Since for each $x \in D$, we have

$$
\int_{D} G_{D}(x, y) v_{k}(y)|q(y)| d y \leq \alpha_{q} v_{k}(x)
$$

the result follows from the monotone convergence theorem.

Proposition 2.3 (see [1]). Let $q$ be a function in $K^{\infty}(D)$. Then

(a) the potential $V q$ is bounded in $D$ and $\lim _{x \rightarrow z \in \partial D} V q(x)=0$,

(b) the function $x \rightarrow\left(\delta_{D}(x) /|x|^{n-1}\right) q(x)$ is in $L^{1}(D)$,

(c)

$$
\theta(x) \preceq V q(x)
$$

Proposition 2.4 (see [1]). Let $q$ be a nonnegative function in $K^{\infty}(D)$. Then the family of function

$$
\mathscr{F}_{q}=\{V p ; p \leq q\}
$$

is relatively compact in $C_{0}(D)$.

Example 2.5. Let $p>n / 2$ and $\lambda, \mu \in \mathbb{R}$ such that $\lambda<2-n / p<\mu$. Then using the Hölder inequality and the same arguments as in ([1, Proposition 3.4]), we prove that for each $f \in$ $L^{p}(D)$, the function defined on $D$ by $f(x) /|x|^{\mu-\lambda}\left(\delta_{D}(x)\right)^{\lambda}$ belongs to $K^{\infty}(D)$. Moreover, by taking $p=+\infty$, we find again the results of [1]. 
8 Positive solutions on some exterior domains

Proposition 2.6. Let $v$ be a nonnegative superharmonic function in $D$ and $q \in K_{+}^{\infty}(D)$. Then for each $x \in D$ such that $0<v(x)<\infty$, we have

$$
\exp \left(-\alpha_{q}\right) \cdot v(x) \leq v(x)-V_{q}(q v)(x) \leq v(x)
$$

Proof. Let $v$ be a nonnegative superharmonic function in $D$. Then by [13, Theorem 2.1, page 164], there exists a sequence $\left(f_{k}\right)_{k}$ of positive measurable functions in $D$ such that the sequence $\left(v_{k}\right)_{k}$ given in $D$ by

$$
v_{k}(x):=\int_{D} G_{D}(x, y) f_{k}(y) d y
$$

increases to $v$.

Let $x \in D$ such that $0<v(x)<\infty$. Then there exists $k_{0} \in \mathbb{N}$ such that $0<V f_{k}(x)<\infty$, for $k \geq k_{0}$.

Now, for a fixed $k \geq k_{0}$, we consider the function $\gamma(t)=V_{t q} f_{k}(x)$.

Since by (viii) the function $\gamma$ is completely monotone on $[0, \infty)$, then $\log \gamma$ is convex on $[0, \infty)$.

Therefore

$$
\gamma(0) \leq \gamma(1) \exp \left(-\frac{\gamma^{\prime}(0)}{\gamma(0)}\right)
$$

which means

$$
V f_{k}(x) \leq V_{q} f_{k}(x) \exp \left(\frac{V\left(q V f_{k}\right)(x)}{V f_{k}(x)}\right)
$$

Hence, it follows from Proposition 2.3 that

$$
\exp \left(-\alpha_{q}\right) \cdot V f_{k}(x) \leq V_{q} f_{k}(x) .
$$

Consequently, from (1.24) we obtain that

$$
\exp \left(-\alpha_{q}\right) \cdot V f_{k}(x) \leq V f_{k}(x)-V_{q}\left(q V f_{k}(x)\right)(x) \leq V f_{k}(x) .
$$

By letting $k \rightarrow \infty$, we deduce the result.

\section{First existence result}

In this section, we give an existence result for problem (1.8). We recall that $\theta(x)=\delta_{D}(x) /$ $\left((1+|x|)^{n-1}\right) \sim \delta_{D}(x) /|x|^{n-1}$ and we prove Theorem 1.2.

Proof of Theorem 1.2. Assuming $\left(\mathrm{H}_{1}\right)-\left(\mathrm{H}_{3}\right)$, we will use the Schauder fixed point theorem. Let $K$ be a compact of $D$ such that we have

$$
0<\alpha:=\int_{K} \theta(y) p(y) d y<\infty,
$$

where $p$ is given in $\left(\mathrm{H}_{3}\right)$. 
We put $\beta:=\min \{\theta(x): x \in K\}$. We note that by (2.4) there exists a constant $\alpha_{1}>0$ such that for each $x, y \in D$

$$
\alpha_{1} \theta(x) \theta(y) \leq G_{D}(x, y)
$$

Then from (1.10), we deduce that there exists $a>0$ such that

$$
\alpha_{1} \alpha f(a \beta) \geq a
$$

On the other hand, since $q \in K^{\infty}(D)$, then by Proposition 2.4 we have that $\|V q\|_{\infty}<\infty$. So taking $\limsup \sup _{t \rightarrow \infty} g(t) / t<\delta<1 /\|V q\|_{\infty}$ we deduce by (1.11) that there exists $\rho>0$ such that for $t \geq \rho$ we have $g(t) \leq \delta t$. Put $\gamma=\sup _{0 \leq t \leq \rho} g(t)$. So we have that

$$
0 \leq g(t) \leq \delta t+\gamma ; \quad t \geq 0
$$

Furthermore by (2.13), we note that there exists a constant $\alpha_{2}>0$ such that

$$
\alpha_{2} \theta(x) \leq V q(x) ; \quad \forall x \in D
$$

and from $\left(\mathrm{H}_{2}\right)$ and Proposition 2.4, we have $\|V \varphi(\cdot, a \theta)\|_{\infty}<\infty$.

Let $b=\max \left\{a / \alpha_{2},\left(\delta\|V \varphi(\cdot, a \theta)\|_{\infty}+\gamma\right) /\left(1-\delta\|V q\|_{\infty}\right)\right\}$ and consider the closed convex set

$$
\Lambda=\left\{u \in C_{0}(D): a \theta(x) \leq u(x) \leq V \varphi(\cdot, a \theta)(x)+b V q(x) ; \forall x \in D\right\}
$$

Obviously, by (3.5) we have that the set $\Lambda$ is nonempty. Define the integral operator $T$ on $\Lambda$ by

$$
T u(x)=\int_{D} G_{D}(x, y)[\varphi(y, u(y))+\psi(y, u(y))] d y ; \quad \forall x \in D .
$$

Let us prove that $T \Lambda \subset \Lambda$. Let $u \in \Lambda$ and $x \in D$, then by (3.4) we have

$$
\begin{aligned}
T u(x) & \leq V \varphi(\cdot, a \theta)(x)+\int_{D} G_{D}(x, y) q(y) g(y) d y \\
& \leq V \varphi(\cdot, a \theta)(x)+\int_{D} G_{D}(x, y) q(y)[\delta u(y)+\gamma] d y \\
& \leq V \varphi(\cdot, a \theta)(x)+\int_{D} G_{D}(x, y) q(y)\left[\delta\left(\|V \varphi(\cdot, a \theta)\|_{\infty}+b\|V q\|_{\infty}\right)+\gamma\right] d y \\
& \leq V \varphi(\cdot, a \theta)(x)+b V q(x) .
\end{aligned}
$$


Moreover from the monotonicity of $f,(3.2)$ and (3.3), we have

$$
\begin{aligned}
T u(x) & \geq \int_{D} G_{D}(x, y) \psi(y, u(y)) d y \\
& \geq \alpha_{1} \theta(x) \int_{D} \theta(y) p(y) f(a \theta(y)) d y \\
& \geq \alpha_{1} \theta(x) f(a \beta) \int_{K} \theta(y) p(y) d y \\
& \geq \alpha_{1} \alpha f(a \beta) \theta(x) \\
& \geq a \theta(x) .
\end{aligned}
$$

On the other hand, we have that for $u \in \Lambda$,

$$
\varphi(\cdot, u) \leq \varphi(\cdot, a \theta), \quad \psi(\cdot, u) \leq\left[\delta\left(\|V \varphi(\cdot, a \theta)\|_{\infty}+b\|V q\|_{\infty}\right)+\gamma\right] q .
$$

This implies by Proposition 2.6 that $T \Lambda$ is relatively compact in $C_{0}(D)$. In particular, we deduce that $T \Lambda \subset \Lambda$.

Next we prove the continuity of $T$ in $\Lambda$. Let $\left(u_{k}\right)_{k}$ be a sequence in $\Lambda$ which converges uniformly to a function $u$ in $\Lambda$. Then since $\varphi$ and $\psi$ are continuous with respect to the second variable, we deduce by the dominated convergence theorem that

$$
\forall x \in D, \quad T u_{k}(x) \longrightarrow T u(x) \text { as } k \longrightarrow \infty .
$$

Now, since $T \Lambda$ is relatively compact in $C_{0}(D)$, then we have the uniform convergence. Hence $T$ is a compact operator mapping $\Lambda$ to itself. So the Schauder fixed point theorem yields to the existence of a function $u \in \Lambda$ such that

$$
u(x)=\int_{D} G_{D}(x, y)[\varphi(y, u(y))+\psi(y, u(y))] d y ; \quad \forall x \in D
$$

Finally since $q$ and $\varphi(\cdot, a \theta)$ are in $K^{\infty}(D)$, we deduce by (3.10) and Proposition 2.4, that the map $y \rightarrow \varphi(y, u(y))+\psi(y, u(y)) \in L_{\text {loc }}^{1}(D)$. Moreover, since $u \in C_{0}(D)$, we deduce from (3.12) that $V(\varphi(\cdot, u)+\psi(\cdot, u)) \in L_{\text {loc }}^{1}(D)$.

Hence $u$ satisfies in the sense of distributions the elliptic equation

$$
\Delta u+\varphi(\cdot, u)+\psi(\cdot, u)=0, \quad \text { in } D
$$

and so it is a solution of the problem (1.8).

Example 3.1. Let $\alpha, \beta \geq 0$ such that $0 \leq \alpha+\beta<1, \gamma>0$ and $p \in K^{\infty}(D)$. Then the problem

$$
\begin{gathered}
\Delta u+p(x)\left[(u(x))^{-\gamma}(\theta(x))^{\gamma}+(u(x))^{\alpha} \log \left(1+(u(x))^{\beta}\right)\right]=0, \quad \text { in } D \\
u>0 \quad \text { in } D
\end{gathered}
$$

has a solution $u \in C_{0}(D)$ satisfying

$$
a \theta(x) \leq u(x) \leq b V p(x),
$$

where $a, b$ are two positive constants. 


\section{Second existence result}

In this section, we aim at proving Theorem 1.3. The proof is based on the following lemma related to the maximum principle for elliptic equation.

For $u \in C(D)$, put $u^{+}=\max (u, 0)$.

Lemma 4.1. Let $\varphi_{1}$ and $\varphi_{2}$ satisfying $\left(H_{4}\right)-\left(H_{6}\right)$. Assume that $\varphi_{1} \leq \varphi_{2}$ on $D \times \mathbb{R}_{+}$and there exist two continuous functions $u, v$ on $D$ satisfying

(a) $\Delta u-\varphi_{1}\left(\cdot, u^{+}\right)=0=\Delta v-\varphi_{2}\left(\cdot, v^{+}\right)$in $D$;

(b) $u, v \in C_{b}(D)$;

(c) $u \geq v$ on $\partial D$ and $\lim _{|x| \rightarrow \infty} u(x) \geq \lim _{|x| \rightarrow \infty} v(x)$.

Then $u \geq v$ in $\bar{D}$.

Proof. Suppose that the open set $\Omega=\{x \in D: u(x)<v(x)\}$ is nonempty. Put $z=u-v$. Then $z \in C_{b}(D)$ and satisfies

$$
\begin{gathered}
\Delta z=\varphi_{1}\left(\cdot, u^{+}\right)-\varphi_{2}\left(\cdot, v^{+}\right) \\
=\left(\varphi_{1}\left(\cdot, u^{+}\right)-\varphi_{2}\left(\cdot, u^{+}\right)\right)+\left(\varphi_{2}\left(\cdot, u^{+}\right)-\varphi_{2}\left(\cdot, v^{+}\right)\right) \leq 0 \quad \text { in } \Omega \\
z \geq 0 \quad \text { on } \partial \Omega \\
\lim _{|x| \rightarrow \infty, x \in \Omega} z(x) \geq 0 .
\end{gathered}
$$

Hence from ([4, page 420]), we conclude that $z \geq 0$ in $\Omega$, which is in contradiction with the definition of $\Omega$. This completes the proof.

Proof of Theorem 1.3. An immediate consequence of the comparison principle, given by Lemma 4.1, is that the problem (1.15) has at most one solution in $D$. The existence of such a solution is assured by the Schauder fixed point theorem. Indeed, to construct a solution, we consider the convex set

$$
\Lambda=\left\{u \in C_{b}(D): u \leq c\right\}
$$

where $c:=\lambda+\|\xi\|_{\infty}$.

We define the integral operator $T$ on $\Lambda$ by

$$
T u(x)=\lambda h(x)+H \xi(x)-V \varphi\left(\cdot, u^{+}\right)(x) ; \quad \text { for } x \in D,
$$

where $h$ is given by (1.17).

Since $\|H \xi\|_{\infty} \leq\|\xi\|_{\infty}$, then for each $u \in \Lambda$, we have

$$
T u(x) \leq \lambda h(x)+H \xi(x) \leq \lambda+\|\xi\|_{\infty}=c ; \quad \text { for each } x \in D .
$$

Furthermore, putting $q=\varphi(\cdot, c)$, we have by $\left(\mathrm{H}_{6}\right)$ that $q \in K^{\infty}(D)$. So by $\left(\mathrm{H}_{4}\right)$, we deduce that $V \varphi\left(\cdot, u^{+}\right) \in \mathscr{F}_{q}$. This together with the fact that $h$ and $H \xi$ are in $C_{b}(D)$ imply by Proposition 2.4 that $T \Lambda$ is relatively compact in $C_{b}(D)$ and in particular $T \Lambda \subset \Lambda$.

From the continuity of $\varphi$ with respect to the second variable, we deduce that $T$ is continuous in $\Lambda$ and so it is a compact operator from $\Lambda$ to itself. Then by the Schauder fixed point theorem, we deduce that there exists a function $u \in \Lambda$ satisfying

$$
u(x)=\lambda h(x)+H \xi(x)-V \varphi\left(\cdot, u^{+}\right)(x) .
$$


This implies, using Proposition 2.4 and the fact that $V \varphi\left(\cdot, u^{+}\right) \in C_{0}(D)$, that $u$ satisfies in the sense of distributions

$$
\begin{gathered}
\Delta u-\varphi\left(\cdot, u^{+}\right)=0 \quad \text { in } D, \\
u=\xi \quad \text { on } \partial D, \\
\lim _{|x| \rightarrow \infty} u(x)=\lambda .
\end{gathered}
$$

Therefore using hypothesis $\left(\mathrm{H}_{5}\right)$ and Lemma 4.1 we deduce that $u \geq 0$.

Corollary 4.2. Let $\varphi$ satisfying $\left(H_{4}\right)-\left(H_{6}\right)$, $\xi$ be a nontrivial nonnegative continuous function on $\partial D$ and $\lambda \geq 0$. Suppose that there exists a function $q \in K^{\infty}(D)$ such that

$$
0 \leq \varphi(x, t) \leq q(x) t \quad \text { on } D \times\left[0, \lambda+\|\xi\|_{\infty}\right] .
$$

Then the solution $u$ of (1.15) given in Theorem 1.3 satisfies

$$
e^{-\alpha_{q}}(\lambda h(x)+H \xi(x)) \leq u(x) \leq \lambda h(x)+H \xi(x) .
$$

Proof. Let $\omega(x)=\lambda h(x)+H \xi(x)$. Since $u$ satisfies the integral equation

$$
u(x)=\omega(x)-V \varphi(\cdot, u)(x),
$$

then using (1.24), we obtain

$$
\begin{aligned}
u-V_{q}(q u) & =\omega-V_{q}(q \omega)-\left[V \varphi(\cdot, u)-V_{q}(q V(\varphi(\cdot, u)))\right] \\
& =\omega-V_{q}(q \omega)-V_{q}(\varphi(\cdot, u)) .
\end{aligned}
$$

That is

$$
u=\omega-V_{q}(q \omega)+V(q u-\varphi(\cdot, u)) .
$$

Now since $0<u \leq \lambda+\|\xi\|_{\infty}$ then by (4.7), we conclude the result from Proposition 2.6.

Example 4.3. Let $\xi$ be nontrivial nonnegative continuous function on $\partial D$. Let $\sigma>0$ and $q \in K^{\infty}(D)$. Put $\varphi(x, t)=q(x) t^{\sigma}$. Then for each $\lambda \geq 0$ the following problem:

$$
\begin{aligned}
\Delta u-q(x) u^{\sigma}=0, \quad & \text { in } D(\text { in the sense of distributions), } \\
& u=\xi \quad \text { on } \partial D, \\
& \lim _{|x| \rightarrow \infty} u(x)=\lambda
\end{aligned}
$$

has a positive bounded continuous solution $u$ satisfying in $D$

$$
0 \leq \lambda h(x)+H \xi(x)-u(x) \leq\left(\lambda+\|\xi\|_{\infty}\right)^{\sigma} V q(x) .
$$

In particular if $\sigma>1$, then there exists $c \in(0,1)$ such that

$$
c(\lambda h(x)+H \xi(x)) \leq u(x) \leq \lambda h(x)+H \xi(x) .
$$


Proof of Corollary 1.5. Let $\rho(t)=\int_{0}^{t}\left(e^{\int_{0}^{s} a(r) d r}\right) d s$, for $t \geq 0$. Then $\rho$ is a $\mathscr{C}^{2}$ diffeomorphism from $[0, \infty)$ to itself. Let $v=\rho(u)$. Then $v$ satisfies

$$
\begin{gathered}
\Delta v=\rho^{\prime}\left(\rho^{-1}(v)\right) \varphi\left(y, \rho^{-1}(v)\right) \quad \text { in } D, \\
v=\rho \circ \xi \quad \text { on } \partial D, \\
\lim _{|x| \rightarrow \infty} v(x)=\rho(\lambda) \geq 0 .
\end{gathered}
$$

Put $\phi(y, v)=\rho^{\prime}\left(\rho^{-1}(v)\right) \varphi\left(y, \rho^{-1}(v)\right)$ for $y \in D$. Then $\phi$ satisfies the same hypothesis as $\varphi$. Hence from Theorem 1.3 the problem (4.15) has a unique nontrivial nonnegative bounded solution $v \in C^{2}(D)$. Consequently $u=\rho^{-1}(v)$ is the unique nontrivial nonnegative bounded solution in $C^{2}(D)$ of the problem (1.18).

\section{Acknowledgment}

The author is greatly indebted to Professor H. Mâagli for many helpful suggestions.

\section{References}

[1] I. Bachar, H. Mâagli, and N. Zeddini, Estimates on the Green function and existence of positive solutions of nonlinear singular elliptic equations, Communications in Contemporary Mathematics 5 (2003), no. 3, 401-434.

[2] H. Brezis and S. Kamin, Sublinear elliptic equations in $\mathbb{R}^{n}$, Manuscripta Mathematica 74 (1992), no. $1,87-106$.

[3] K. L. Chung and Z. X. Zhao, From Brownian Motion to Schrödinger's Equation, Grundlehren der Mathematischen Wissenschaften, vol. 312, Springer, Berlin, 1995.

[4] R. Dautray and J.-L. Lions, Analyse mathématique et calcul numérique pour les sciences et les techniques, L' opérateur de Laplace, Coll. C.E.A, vol. 2, Masson, Paris, 1987.

[5] J. I. Diaz, J.-M. Morel, and L. Oswald, An elliptic equation with singular nonlinearity, Communications in Partial Differential Equations 12 (1987), no. 12, 1333-1344.

[6] A. L. Edelson, Entire solutions of singular elliptic equations, Journal of Mathematical Analysis and Applications 139 (1989), no. 2, 523-532.

[7] T. Kusano and C. A. Swanson, Entire positive solutions of singular semilinear elliptic equations, Japanese Journal of Mathematics. New Series 11 (1985), no. 1, 145-155.

[8] A. V. Lair and A. W. Shaker, Classical and weak solutions of a singular semilinear elliptic problem, Journal of Mathematical Analysis and Applications 211 (1997), no. 2, 371-385.

[9] A. C. Lazer and P. J. McKenna, On a singular nonlinear elliptic boundary-value problem, Proceedings of the American Mathematical Society 111 (1991), no. 3, 721-730.

[10] H. Mâagli, Perturbation semi-linéaire des résolvantes et des semi-groupes [Semilinear perturbation of resolvents and semigroups], Potential Analysis 3 (1994), no. 1, 61-87.

[11] H. Mâagli and S. Masmoudi, Positive solutions of some nonlinear elliptic problems in unbounded domain, Annales Academiæ Scientiarium Fennicæ. Mathematica 29 (2004), no. 1, 151-166.

[12] H. Mâagli and M. Zribi, Existence and estimates of solutions for singular nonlinear elliptic problems, Journal of Mathematical Analysis and Applications 263 (2001), no. 2, 522-542.

[13] S. C. Port and C. J. Stone, Brownian Motion and Classical Potential Theory. Probability and Mathematical Statistics, Academic Press, New York, 1978.

Noureddine Zeddini: Département de Mathématiques, Faculté des Sciences de Tunis, Campus Universitaire, 2092 Tunis, Tunisia

E-mail address: noureddine.zeddini@ipein.rnu.tn 


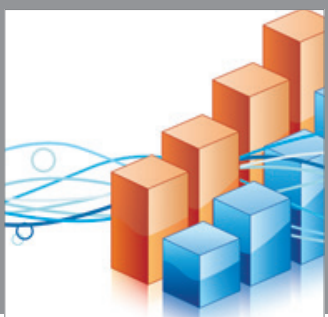

Advances in

Operations Research

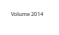

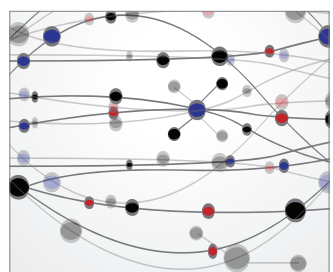

\section{The Scientific} World Journal
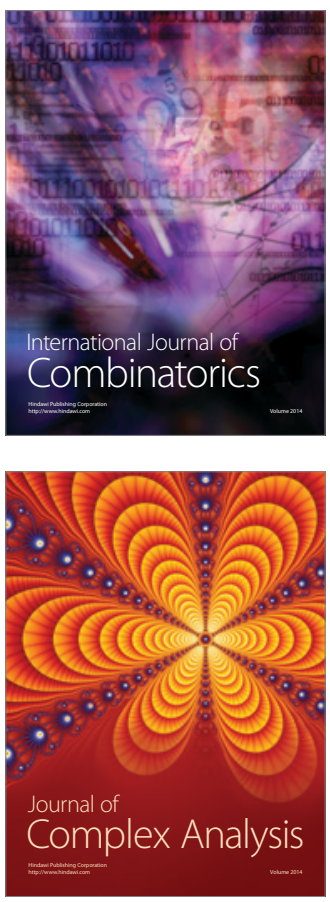

International Journal of

Mathematics and

Mathematical

Sciences
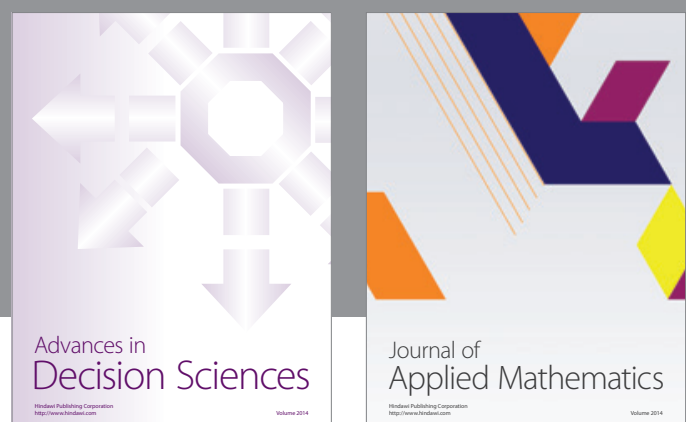

Journal of

Applied Mathematics
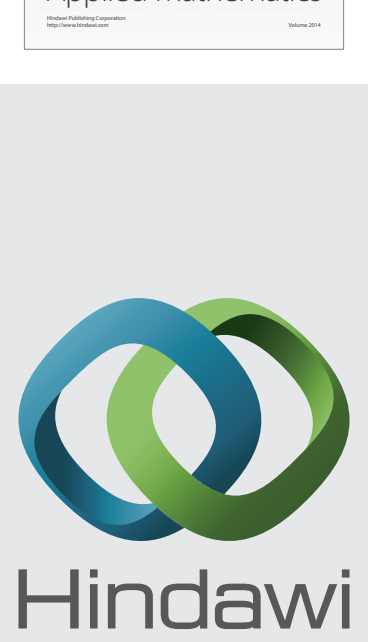

Submit your manuscripts at http://www.hindawi.com
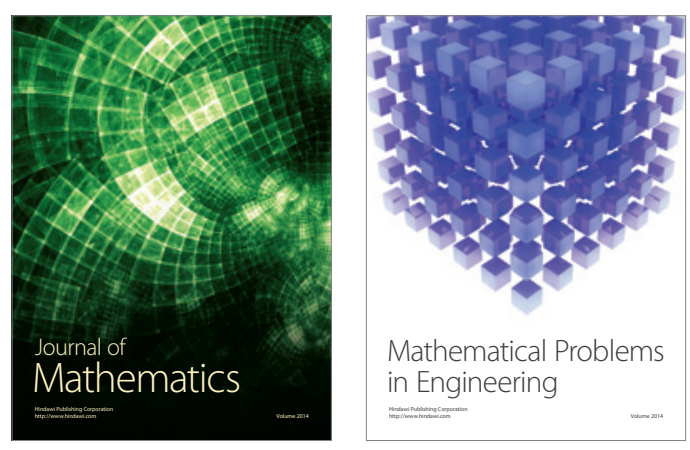

Mathematical Problems in Engineering
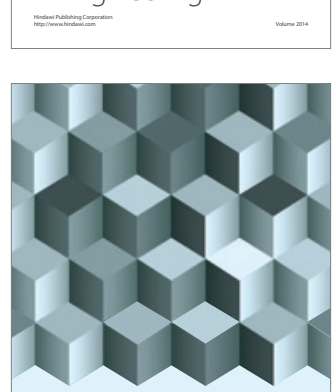

Journal of

Function Spaces
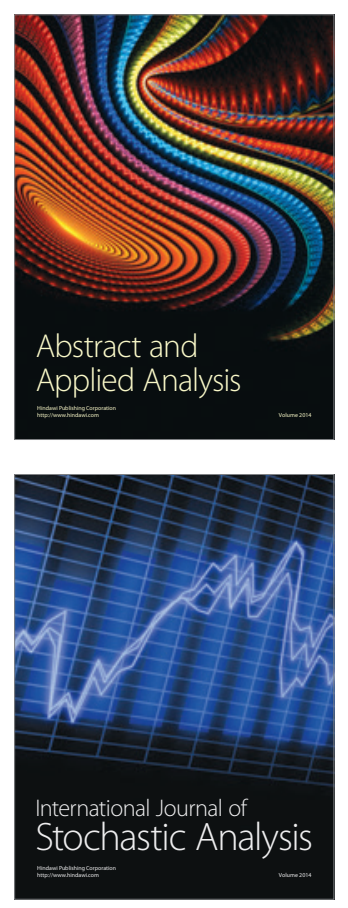

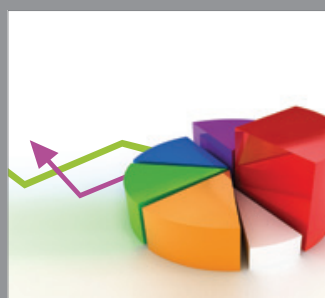

ournal of

Probability and Statistics

Promensencen
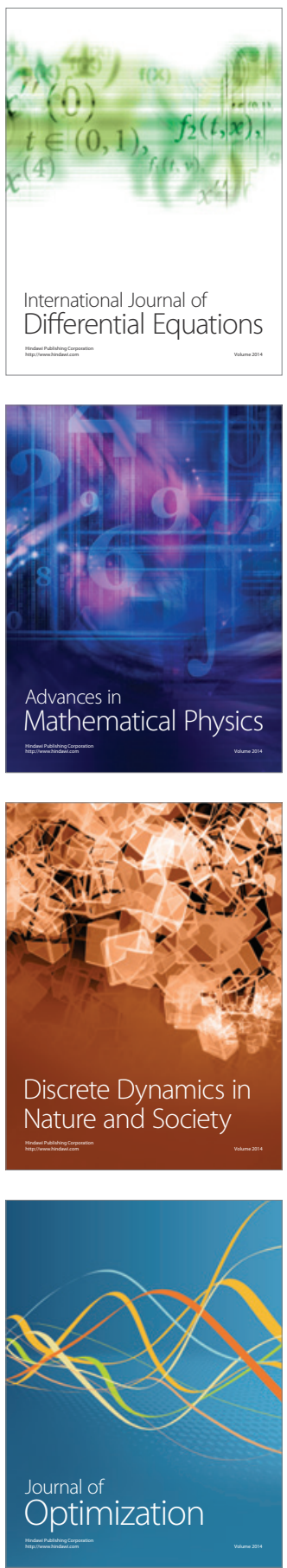